\title{
Preferência hospedeira do parasitoide de ovos Trichogramma pretiosum
}

\author{
Host preference of the egg parasitoid Trichogramma pretiosum
}

\author{
Jullyana Rodrigues Siqueira ${ }^{\mathrm{I}}$ Regiane Cristina Oliveira de Freitas Bueno ${ }^{\mathrm{II}}$ \\ Adeney de Freitas Bueno ${ }^{\text {III }}$ Simone Silva Vieira ${ }^{\text {IV }}$
}

\begin{abstract}
O sucesso das liberações de Trichogramma spp. depende basicamente do conhecimento das características bioecológicas do parasitoide e da sua interação com o hospedeiro alvo. Assim, o objetivo deste trabalho foi avaliar a preferência hospedeira de $\boldsymbol{T}$. pretiosum entre os ovos de $\boldsymbol{S}$. frugiperda, S. albula, S. eridania, A. gemmatalis, $P$. includens, D. saccharalis e A. kuehniella. Para os testes de preferência, foram instaladas arenas com garrafas de polietileno e, em cada uma delas, foi colocada uma cartela com ovos de duas espécies de hospedeiros e uma fêmea do parasitoide por 24 horas. Os parâmetros avaliados foram o parasitismo (\%) e a emergência (\%). As fêmeas de T. pretiosum apresentaram preferência pelos ovos de A. kuehniella. No entanto, nos ensaios com A. gemmatalis $X P$. includens e D. saccharalis $X S$. frugiperda, não ocorreram diferenças no parasitismo. Assim, pode-se afirmar que, nos teste de livre escolha, na ausência do hospedeiro em que os parasitoides foram multiplicados (A. kuehniella), as fêmeas não apresentaram preferência ao parasitismo, o que indica a existência de condicionamento pré-imaginal do parasitoide. Os resultados da viabilidade dos ovos parasitados por T. pretiosum variaram entre os diferentes hospedeiros testados.
\end{abstract}

Palavras-chave: controle biológico, parasitoide de ovos, condicionamento pré-imaginal.

\section{ABSTRACT}

The success of Trichogramma spp. releases in biological control programs relies upon the knowledge of the parasitoid bio-ecological characteristics and the parasitoid interaction with the target pest. Therefore, this research aimed to evaluate $\boldsymbol{T}$. pretiosum host preference among eggs of $\boldsymbol{S}$. frugiperda, S. albula, S. eridania, A. gemmatalis, P. includens, $D$. saccharalis and A. kuehniella. Polyethylene cages were set to test host preference of $\boldsymbol{T}$. pretiosum comparing each two groups of host species. The evaluated parameter was parasitism (\%) and parasitoid emergence (\%). T. pretiosum females parasitized preferably A. kuehniella eggs. However, in the bioassay with A. gemmatalis $X P$. includens and $D$. saccharalis $X S$. frugiperda there was no difference in the parasitism. Then, in the free choice test, in the absence of the host in which the parasitoid was reared (A. kuehniella), T. pretiosum shows no host preference indicating the existence of pre-imaginal conditioning acquired during larval development. The parasitism viability results were variable among the different tested hosts.

Key words: Biological control, egg parasitoid, pre-imaginal conditioning.

Os parasitoides de ovos Trichogramma spp. apresentam potencial de utilização em programas de controle biológico de diversos lepidópteros-praga em várias culturas de importância econômica, por apresentarem ampla distribuição geográfica, ter a capacidade de parasitar ovos de diferentes hospedeiros, além da facilidade da multiplicação dos insetos em ovos de hospedeiros alternativos. Tais características podem influenciar no sucesso das liberações de Trichogramma spp., que é baseado no conhecimento das características bioecológicas do parasitoide e da sua interação com o hospedeiro alvo.

'Universidade Católica de Goiás (UCG), Setor Universitário, Goiânia, GO, Brasil.

"Universidade de Rio Verde (FESURV), Rio Verde, GO, Brasil.

IIEmbrapa Soja, CP 231, 86001-970, Londrina, PR, Brasil. E-mail: adeney@cnpso.embrapa.br. Autor para correspondência.

${ }^{\mathrm{IV}}$ Instituto Agronômico de Campinas (IAC), Campinas, SP, Brasil. 
Portanto, estudos que antecedam a liberação dos parasitoides devem ser realizados, avaliando-se a capacidade de busca dos ovos, a preferência hospedeira e a tolerância às condições climáticas (PARRA\& ZUCCHI, 2004).

O conhecimento da preferência dos parasitoides é importante porque em campo existe a ocorrência de mais de uma espécie de inseto, sendo que esta diversidade pode interferir no desempenho do parasitoide em suprimir o número de insetos de uma praga-alvo, em virtude da maior oferta de ovos. Outro ponto importante a ser considerado é a qualidade do parasitoide multiplicado em criações massais, pois esta é realizada em ovos do hospedeiro alternativo Anagasta kuehniella (Zeller, 1879) (Lepidoptera: Pyralidae) durante sucessivas gerações, rotina que pode afetar a preferência, alterando a eficiência do parasitismo(COBERT, 1985).

Diante disso, o objetivo deste trabalho foi avaliar a preferência de Trichogramma pretiosum Riley, 1879 (Hymenoptera: Trichogrammatidae) em relação às pragas Spodoptera frugiperda (J.E. Smith, 1797), Spodoptera albula (Walker, 1857), Spodoptera eridania (Cramer, 1782), Anticarsia gemmatalis Hübner, 1818, Pseudoplusia includens (Walker, 1857) (Lepidoptera: Noctuidae) e Diatraea saccharalis (Fabricius, 1794) (Lepidoptera: Crambidae), que ocorrem em culturas com importância econômica como o milho, o algodão, a soja e a cana-de-açúcar e o hospedeiro alternativo a A. kuehniella, cujo parasitoide vem sendo criado por várias gerações.

Os ensaios foram conduzidos em laboratório sob condições controladas de $25 \pm 2^{\circ} \mathrm{C}$ e $70 \pm 10 \%$ e fotofase de 14 horas em delineamento inteiramente casualizado com 2 tratamentos e 12 repetições em cada bioensaio, visando a avaliar a preferência hospedeira de T. pretiosum em diferentes hospedeiros comparados em teste de dupla escolha. Os hospedeiros naturais foram criados em dietas artificiais de acordo com PARRA(2001) e o hospedeiro alternativo foi criado conforme a metodologia proposta por PARRA et al. (1989). A criação de $T$. pretiosum foi mantida segundo a metodologia descrita por STEIN \& PARRA (1987) em ovos de hospedeiro alternativo A. kuehniella. Os insetos foram mantidos em salas climatizadas, com temperatura de $25 \pm 1^{\circ} \mathrm{C}$, UR $70 \pm 10 \%$ e fotofase de 14 horas.

Os testes de preferência hospedeira foram conduzidos com chance de escolha em arenas propostas por THULER et al. (2007). Cada arena foi composta por garrafas transparentes de polietileno de $4 \mathrm{~cm}$ de altura e $2 \mathrm{~cm}$ de diâmetro, com 4 microtubos de plástico $(1,5 \mathrm{~mL})$ dispostos equidistantes entre si, contendo em cada tubo uma cartela de cartolina de $0,9 \times 6,0 \mathrm{~cm}$ com aproximadamente 20 ovos dos hospedeiros, os quais foram oferecidos para cada fêmea de T. pretiosum de forma alternada na arena e um quinto tubo com uma fêmea do parasitoide foi colocado no centro da arena de forma vertical, possibilitando a livre escolha do parasitoide entre os ovos dos diferentes hospedeiros oferecidos. Os ovos com até 24 horas foram expostos ao parasitismo por um período de 24 horas e posteriormente as fêmeas, também com até 24 horas, foram retiradas dos tubos e os ovos foram mantidos nas mesmas condições controladas nas quais foram conduzidos os ensaios e diariamente foram observados até a emergência dos parasitoides, quando foi então realizada a avaliação do parasitismo. Nos ensaios realizados com $\boldsymbol{T}$. pretiosum, as comparações de hospedeiros avaliadas foram: A. kuehniella X $\boldsymbol{A}$. gemmatalis, A. kuehniella X D. saccharalis, A. kuehniella X P. includens, A. kuehniella X S. albula, A. kuehniella X S. cosmioides, A. kuehniella X S. eridania, A. kuehniella X S. frugiperda, A. gemmatalis X P. includens, e D. saccharalis X $S$. frugiperda. Os parâmetros avaliados foram o parasitismo (\%) e emergência (\%) em cada hospedeiro comparado dois a dois e os resultados obtidos foram submetidos às análises exploratórias para avaliar as pressuposições de normalidade dos resíduos, a homogeneidade de variância dos tratamentos e a aditividade do modelo para permitir a aplicação da ANOVA e assim a comparação das médias pelo teste $\mathrm{F}$ a $5 \%$ de probabilidade.

As fêmeas de $\boldsymbol{T}$. pretiosum avaliadas no teste de escolha com ovos das diferentes espécies de hospedeiros apresentaram preferência para o parasitismo por A. kuehniella, que foi o hospedeiro em que o parasitoide é multiplicado em laboratório (Figura 1).

A preferência de $\boldsymbol{T}$. pretiosum a ovos de A. kuehniella foi confirmada pelo maior parasitismo $(83,34 ; 81,80 ; 74,28 \%$, respectivamente) em ovos deste hospedeiro em relação a $\boldsymbol{A}$. gemmatalis $(16,66 \%)$ (Figura 1A), D. saccharalis $(18,20 \%)$ (Figura 1B), P. includens $(25,72 \%)$ (Figura 1C). Nas espécies de Spodoptera spp. testadas (Figuras 1D, 1E, 1F e 1G), o parasitismo também foi menor, com valores entre 0,84 a 20,72\% contra 77,5 a 94,5\% em A. kuehniella (Figuras $1 \mathrm{D}, 1 \mathrm{E}, 1 \mathrm{~F}$ e 1G). Não houve preferência do parasitismo de $\boldsymbol{T}$. pretiosum entre os hospedeiros A. gemmatalis X P. includens e D. saccharalis X S. frugiperda (Figuras 1He 1I). Assim, pode-se afirmar que, nos teste de livre escolha, na ausência do hospedeiro em que os parasitoides são mantidos em criação no laboratório, 


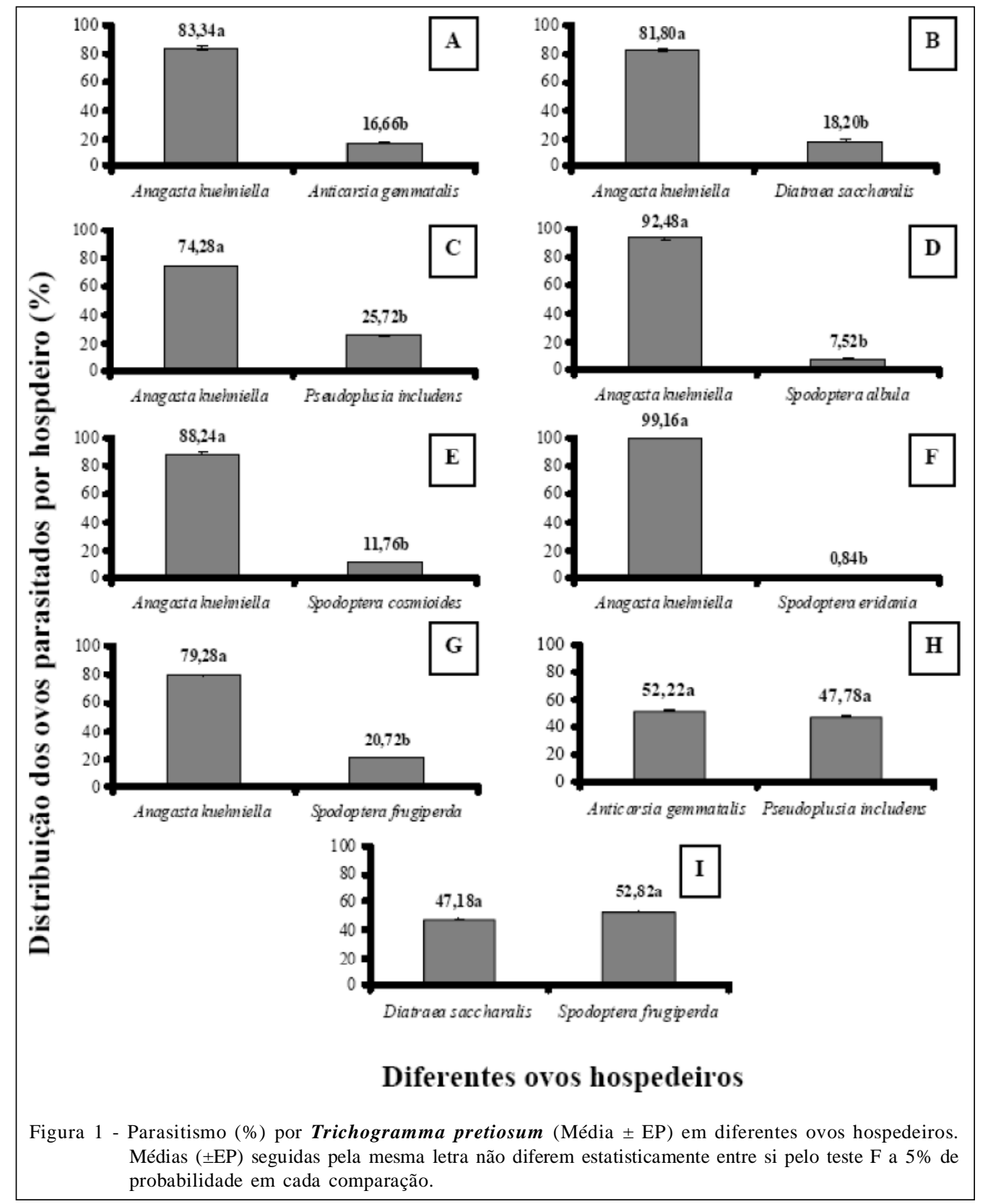

as fêmeas não apresentaram preferência ao parasitismo por um determinado hospedeiro.

A preferência para o parasitismo em ovos de A. kuehniella por T. pretiosum indica a existência de condicionamento pré-imaginal do parasitoide, adquirido durante o desenvolvimento larval, o que, segundo COBERT (1985), pode ocorrer quando um parasitoide é criado por várias gerações no hospedeiro alternativo. Entretanto, a preferência das fêmeas de Trichogramma spp. tem relações complexas com a qualidade nutricional de cada hospedeiro que está sendo escolhido para o parasitismo e também em relação ao hospedeiro em que o parasitoide foi multiplicado (THULER et al., 2007). Entre as diferenças que podem ser destacadas nos hospedeiros, incluemse diferenças nas superfícies, tamanho, estruturas do córion de cada ovo hospedeiro e também as mudanças na cor durante o desenvolvimento embrionário, tamanho e volume do ovo. Todas essas peculiaridades específicas de cada ovo, assim como as diferenças relativas a cada hospedeiro podem influenciar não apenas no tempo de manuseio e exploração por 
Trichogramma spp., mas também na suscetibilidade do hospedeiro, assim como no desenvolvimento do parasitoide (CÔNSOLI et al., 1999).

Os resultados da viabilidade dos ovos parasitados por $\boldsymbol{T}$. pretiosum variaram entre os diferentes hospedeiros testados (Figuras $2 \mathrm{~A}$ a I). A emergência (\%) de adultos provenientes dos ovos do hospedeiro alternativo A. kuehniella parasitados por T. pretiosum foi sempre elevada, como valores superiores a 77,50\% independente do hospedeiro ao qual foi comparado (Figuras 2A, 2B, 2C, 2D, 2E, 2F, e 2G). Nos ovos dos hospedeiros A. gemmatalis, $\boldsymbol{P}$. includens e S. frugiperda, a emergência (\%) dos adultos também foi favorável com valores superiores a $85 \%$ (Figuras $2 \mathrm{He} \mathrm{I}$ ).

A avaliação da emergência (\%) de adultos complementa os resultados da avaliação de preferência, pois mostra se o hospedeiro é suficientemente satisfatório para o parasitoide completar seu desenvolvimento. Independentemente do parasitismo, baixas taxas de emergência podem prejudicar programas de controle biológico. Valores da viabilidade (\%) do parasitismo de T. pretiosum são relatados na literatura em ovos de $\boldsymbol{S}$. frugiperda de 95,37\% (BESERRA \& PARRA, 2004), em ovos de A. kuehniella e Helicoverpa zea (Boddie, 1850) (Lepidoptera:

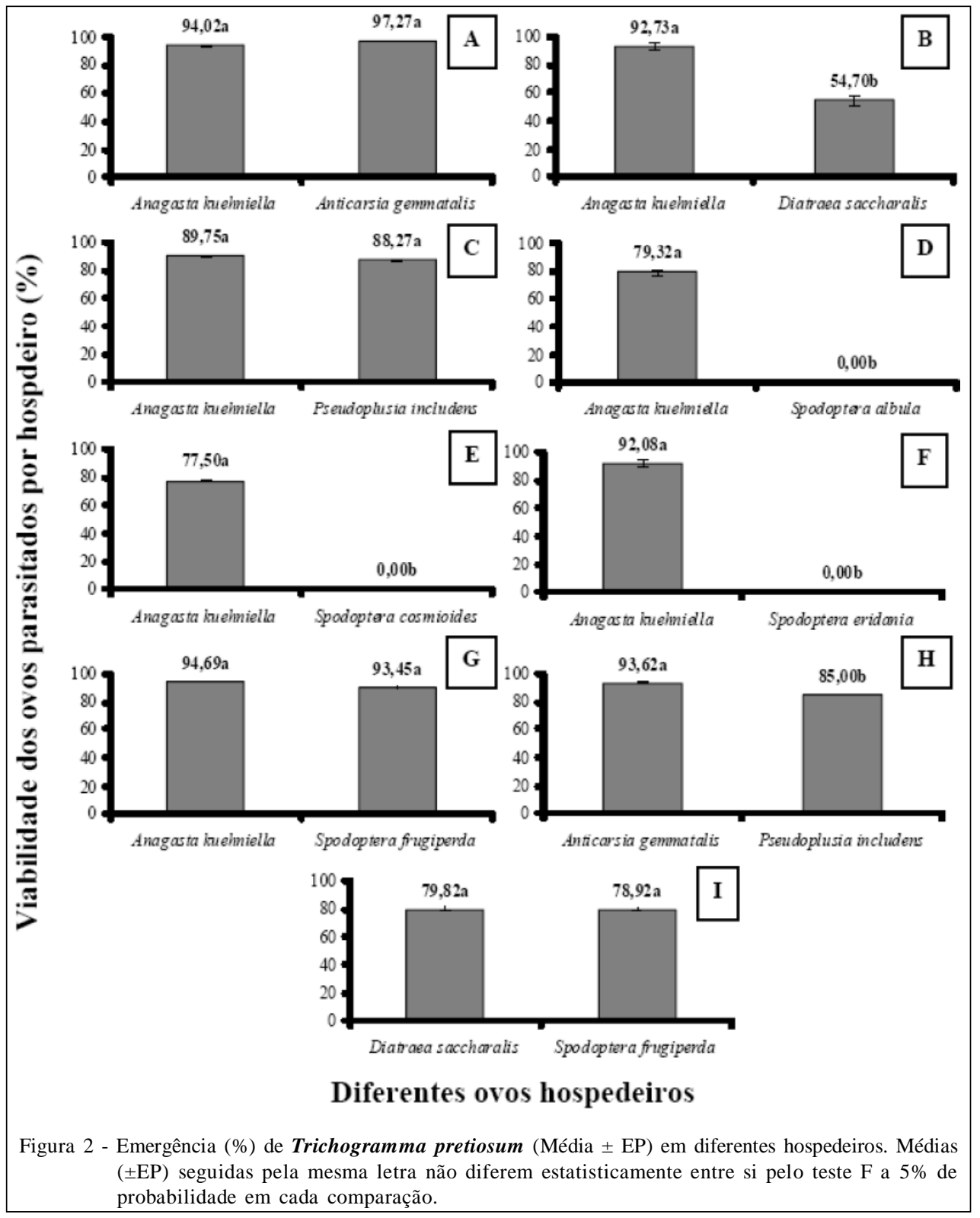

Ciência Rural, v.42, n.1, jan, 2012. 
Noctuidae) de 86,66 e 95,00\%, respectivamente (SÁ \& PARRA, 1994), indicando que emergência de adultos com valores próximos a $80 \%$ são os relacionados a esta espécie de parasitoide. No entanto, a emergência (\%) de espécies/linhagens de Trichogramma em ovos de Gymnandrosoma aurantianum Lima, 1927 (Lepidoptera Tortricidae) pode variar de 30,9 a 42,1\% (MOLINA et al., 2005) em razão da perda de umidade deles pela existência de grande número de aerópilas presentes no córion (MOLINA et al., 2005; CÔNSOLI et al., 1999), o que pode explicar a diferença na viabilidade de T. pretiosum em ovos de D. saccharalis, pois esta espécie também apresenta aerópilas em alta concentração distribuídas na superfície do ovo (CÔNSOLI et al., 1999).

Por outro lado, é importante salientar que a emergência de $\boldsymbol{T}$. pretiosum em ovos de $\boldsymbol{S}$. albula, $\boldsymbol{S}$. eridania e S. cosmioides foi inexistente (Figuras 2 D, E e F), o que demonstra que nos poucos ovos parasitados não ocorreu o desenvolvimento da progênie. Este estudo é um dos pioneiros em avaliar o parasitismo de T. pretiosum em ovos dessas espécies de Spodoptera, demonstrando que, com base nos resultados, esses hospedeiros não proporcionam desenvolvimento satisfatório dos parasitoides, mas novos estudos ainda precisam ser realizados para investigar as razões para a não preferência e a inexistência da emergência de adultos nessas espécies.

\section{AGRADECIMENTOS}

A Embrapa Soja pelo apoio na realização da pesquisa. A Coordenação de Aperfeiçoamento de Pessoal de Nível Superior (CAPES) - processo 23038.035744/2008-89 - pelo apoio financeiro e concessão das bolsas que tornaram viáveis a realização deste trabalho. Este artigo foi revisado e aprovado para publicação pelo Comitê de Publicações da Embrapa Soja.

\section{REFERÊNCIAS}

BESERRA, E.B.; PARRA, J.R.P. Biologia e parasitismo de Trichogramma atopovirilia Oatman \& Plantner e Trichogramma pretiosum Riley (Hymenoptera: Trichogrammatidae) em ovos de Spodoptera frugiperda (J. E. Smith) (Lepidoptera: Noctuidae). Revista Brasileira de Entomologia, v.48, n.1, p.119-126, 2004. Disponível em: <http://www.scielo.br/scielo.php?script=sci_abstract\&pid=S0085$56262004000100020 \& \operatorname{lng}=\mathrm{pt} \& \mathrm{nrm}=\mathrm{iso} \& \operatorname{lng}=\mathrm{pt}>$. Acesso em: 13 jan. 2011.
COBERT, S.A. Insect chemosensory responses: a chemical legacy hypothesis. Ecological Entomology, v.10, p.143-153, 1985. Disponível em: <http://dx.doi.org/10.1111/j.13652311.1985.tb00543.x>. Acesso em: 12 jan. 2010. doi: 10.1111/ j.1365-2311.1985.tb00543.x.

CÔNSOLI, F.L. et al. Ultrastructure of the natural and factitious host eggs of Trichogramma galloi Zucchi and Trichogramma pretiosum Riley (Hymenoptera: Trichogrammatidae). International Journal of Insect Morphology and Embryology, v.28, p.211-229, 1999. Disponível em: <http:/ /dx.doi.org/10.1016/S0020-7322(99)00026-4>. Acesso em: 23 maio, 2005. doi: 10.1016/S0020-7322(99)00026-4

MOLINA, R.M.S. et al. Seleção de Trichogramma spp., para o controle de Ecdytolopha aurantiana, com base na biologia e exigências térmicas. Revista Brasileira de Entomologia, v.49, n.1, p.152-158, 2005. Disponível em: <http://dx.doi.org/ 10.1590/S0085-56262005000100018>. Acesso em: 22 jun. 2010. doi: $10.1590 / \mathrm{S} 0085-56262005000100018$.

PARRA, J.R.P. et al. Metodologia de criação de Anagasta kuehniella Zeller, 1879 para a produção massal de Trichogramma sp. Anais da Sociedade Entomológica do Brasil, v.18, n.2, p.403-415, 1989.

PARRA, J.R.P. Técnicas de criação de insetos para programa de controle biológico. Piracicaba, SP: FEALQ, 2001. 134p.

PARRA, J.R.P.; ZUCCHI, R.A. Trichogramma in Brazil: feasibility of use after twenty years of research. Neotropical Entomology, v.33, n.3, p.271-281, 2004. Disponível em: <http://dx.doi.org/10.1590/S1519-566X2004000300001>. Acesso em: 12 set. 2010 . doi: 10.1590/S1519566X2004000300001.

SÁ, L.A.N. de; PARRA, J.R.P. Biology and parasitism of Trichogramma pretiosum Riley (Hym.: Pyralidae) on Ephestia kuehniella (Zeller) and Heliothis zea (Boddie) (Lep.: Noctuidae) eggs. Journal of Applied Entomology, v.118, p.38-43, 1994. Disponível em: <http://dx.doi.org/10.1111/ j.1439-0418.1994.tb00776.x>. Acesso em: 22 abr. 2010. doi: 10.1111/j.1439-0418.1994.tb00776.x.

STEIN, C.P.; PARRA, J.R.P. Uso da radiação para inviabilizar os ovos de Anagasta kuehniella visando estudos com Trichogramma sp. Anais da Sociedade Entomológica do Brasil, v.6, n.1, p.229-231, 1987.

THULER R.T. et al. Metodologia para avaliação da preferência hospedeira de parasitoides do gênero Trichogramma Westood. Boletín de Sanidad Vegetal Plagas, v.33, n.3, p.333-340, 2007. Disponível em: <https://www.mapya.es/ministerio/pags/ biblioteca/revistas/pdf_Plagas\%5CBSVP_33_03_333_340.pdf > Acesso em: 22 abr. 2010. 